\title{
A BOUNDEDNESS CRITERION FOR GENERAL MAXIMAL OPERATORS
}

\author{
Andrei K. Lerner And Sheldy Ombrosi
}

\begin{abstract}
We consider maximal operators $M_{\mathcal{B}}$ with respect to a basis $\mathcal{B}$. In the case when $M_{\mathcal{B}}$ satisfies a reversed weak type inequality, we obtain a boundedness criterion for $M_{\mathcal{B}}$ on an arbitrary quasiBanach function space $X$. Being applied to specific $\mathcal{B}$ and $X$ this criterion yields new and short proofs of a number of well-known results. Our principal application is related to an open problem on the boundedness of the two-dimensional one-sided maximal function $M^{+}$on $L_{w}^{p}$.
\end{abstract}

\section{Introduction}

For any point $x \in \mathbb{R}^{n}$ denote by $\mathcal{B}(x)$ a family of bounded measurable sets of positive measure. The unified collection $\mathcal{B}=\cup_{x \in \mathbb{R}^{n}} \mathcal{B}(x)$ is called a basis (see $[\mathbf{8}]$ and also $[\mathbf{9}]$ for a somewhat different definition). For a locally integrable function $f$ on $\mathbb{R}^{n}$ the Hardy-Littlewood maximal operator associated with $\mathcal{B}$ is defined by

$$
M_{\mathcal{B}} f(x)=\sup _{B \in \mathcal{B}(x)} \frac{1}{|B|} \int_{B}|f(y)| d y .
$$

The basis formed by all cubes $Q$ containing $x$ with sides parallel to the axes we denote by $\mathcal{Q}$. If $x=\left(x_{1}, \ldots, x_{n}\right)$ and $\mathcal{B}(x)=\left\{\prod_{i=1}^{n}\left(x_{i}, x_{i}+\right.\right.$ $h)\}_{h>0}$, the corresponding basis is denoted by $\mathcal{Q}^{+}$. The maximal operators associated with $\mathcal{Q}$ and $\mathcal{Q}^{+}$are denoted by $M$ and $M^{+}$, respectively.

The Hardy-Littlewood maximal operator in its various forms plays a fundamental role in harmonic analysis, and its different aspects have been studied in a great number of papers. The most typical problem of interest can be described briefly as follows: given a function space $X$

2000 Mathematics Subject Classification. 42B25.

Key words. Maximal operators, one-sided maximal function.

The authors are supported by a fellowship from the Spanish Ministry of Education. 
and a basis $\mathcal{B}$, find a necessary and sufficient condition yielding the boundedness of $M_{\mathcal{B}}$ on $X$.

Let $M_{\mathcal{B}, r} f=\left(M_{\mathcal{B}}|f|^{r}\right)^{1 / r}$. By Hölder's inequality, $M_{\mathcal{B}, r} f \leq M_{\mathcal{B}, s} f$ if $r<s$. In a recent paper [13], the authors established that $M$ is bounded on a quasi-Banach function space $X$ iff $M_{r}$ is bounded on $X$ for some $r>1$. For many particular spaces $X$ this self-improving phenomenon was observed before but each case required its own proof. In this paper we complement this result by extending it to a wide class of $\mathcal{B}$ and by obtaining a similar characterization in terms of $M_{\mathcal{B}, r}$ for $r<1$. The case $r>1$ in $[\mathbf{1 3}]$ was treated by means of the concept of generalized Boyd indices. Here we give a unified and simple approach to both cases $r>1$ and $r<1$ using the well-known Rubio de Francia algorithm.

The following definition expresses the relevant property of a basis needed for our purposes. In the case when $\mathcal{B}=\mathcal{Q}$ it was obtained by E. M. Stein $[\mathbf{2 3}]$.

Definition 1.1. We say that a basis $\mathcal{B}$ satisfies the Stein property if there exists a constant $c>0$ such that for any $f \in L_{\text {loc }}^{1}\left(\mathbb{R}^{n}\right)$ and $x \in \mathbb{R}^{n}$, for all $B \in \mathcal{B}(x)$ and $\lambda>M_{\mathcal{B}} f(x)$ we have

$$
\int_{\{y \in B:|f(y)|>\lambda\}}|f(y)| d y \leq c \lambda\left|\left\{y \in B: M_{\mathcal{B}} f(y)>\lambda\right\}\right| .
$$

One of our main results is the following.

Theorem 1.2. Let $X\left(\mathbb{R}^{n}\right)$ be an arbitrary quasi-Banach function space. Suppose $\mathcal{B}$ satisfies Stein's property. Then the following conditions are equivalent:

(i) $\lim _{\varepsilon \rightarrow 0} \varepsilon\left\|M_{\mathcal{B}, 1-\varepsilon}\right\|_{X}=0$;

(ii) $M_{\mathcal{B}}$ is bounded on $X$;

(iii) $M_{\mathcal{B}, r}$ is bounded on $X$ for some $r>1$.

In order to get a better feeling for the theorem, let us consider the case when $X$ is the weighted Lebesgue space $L_{w}^{p}$, where a weight $w$ is supposed to be a non-negative locally integrable function. First of all, we have the following.

Corollary 1.3. Let $\mathcal{B}$ satisfy Stein's property, and let $1<p<\infty$. If $M_{\mathcal{B}}$ maps $L_{w}^{p}$ into $L_{w}^{p, \infty}$, then $M_{\mathcal{B}}$ actually maps $L_{w}^{p}$ into $L_{w}^{p}$.

Indeed, if $M_{\mathcal{B}}: L_{w}^{p} \rightarrow L_{w}^{p, \infty}$, then by the Marcinkiewicz interpolation theorem (see, e.g., $\left[\mathbf{5}\right.$, p. 29]), $\left\|M_{\mathcal{B}}\right\|_{L_{w}^{q}} \leq c(q-p)^{-1 / q}$ for $q>p$. Taking $q=\frac{p}{1-\varepsilon}$, we get $\left\|M_{\mathcal{B}, 1-\varepsilon}\right\|_{L_{w}^{p}} \leq c \varepsilon^{-1 / p}$. It remains to apply (i) $\Rightarrow$ (ii). 
Corollary 1.3 shows that in the case when $\mathcal{B}$ satisfies Stein's property, the weak type $(p, p)$ (with respect to $w$ ) of $M_{\mathcal{B}}$ is equivalent to the strong type $(p, p)$ for $p>1$. However, the weak type $(p, p)$ property is usually much easier to prove. Consider, for example, the classical maximal operator $M$. We recall that a weight $w$ satisfies the $A_{p}$ condition if there exists $c>0$ such that for any cube $Q$,

$$
\left(\int_{Q} w\right)\left(\int_{Q} w^{-1 /(p-1)}\right)^{p-1} \leq c|Q|^{p} .
$$

By a fundamental theorem of B. Muckenhoupt [17] (see also [4]), $M$ is bounded on $L_{w}^{p}$ iff $w \in A_{p}$. The first proofs of this result [4], [17] depended on a deep property of $A_{p}$ weights saying that the $A_{p}$ condition implies $A_{p-\varepsilon}$ for some $\varepsilon>0$. Later, other proofs (see, e.g., [9]), avoiding this property, were found. We now observe that Theorem 1.2 implies easily both Muckenhoupt's theorem and the implication $A_{p} \Rightarrow$ $A_{p-\varepsilon}$. Indeed, Hölder's inequality along with the $A_{p}$ condition yields $M f(x)^{p} \leq c M_{w}\left(|f|^{p}\right)(x)$ ( $M_{w}$ is the weighted maximal operator), and since any $A_{p}$ weight is doubling, by a classical covering argument we get the weighted weak type $(p, p)$ of $M$. This, by Corollary 1.3, proves Muckenhoupt's theorem (only the sufficiency part in this theorem is nontrivial). Next, we clearly have that $M_{r}: L_{w}^{p} \rightarrow L_{w}^{p}$ for some $r>1$ iff $M: L_{w}^{p-\varepsilon} \rightarrow L_{w}^{p-\varepsilon}$ for some $\varepsilon>0$. Therefore, by (ii) $\Rightarrow$ (iii) of Theorem 1.2 we get $A_{p} \Rightarrow A_{p-\varepsilon}$.

Consider now the maximal operator $M^{+}$. Given a cube $Q=\prod_{i=1}^{n}\left(a_{i}-\right.$ $\left.h, a_{i}\right)$, set $Q^{+}=\prod_{i=1}^{n}\left(a_{i}, a_{i}+h\right)$. We say that a weight $w$ satisfies the $A_{p}^{+}$condition if there exists $c>0$ such that for any cube $Q$,

$$
\left(\int_{Q} w\right)\left(\int_{Q^{+}} w^{-1 /(p-1)}\right)^{p-1} \leq c|Q|^{p} .
$$

Only fourteen years after Muckenhoupt's result E. Sawyer [21] proved that in the one-dimensional case $M^{+}$is bounded on $L_{w}^{p}$ iff $w \in A_{p}^{+}$. The proof in [21] was based on certain Hardy-type inequalities. Later, F. J. Martín-Reyes [14] found another proof in spirit of the classical case of $M$. Namely, first an equivalence of $A_{p}^{+}$and the weak-type $(p, p)$ of $M^{+}$ was established (which was done in a simple and clever way), and then the property $A_{p}^{+} \Rightarrow A_{p-\varepsilon}^{+}$was proved. Observe that in Sawyer's work [21] it was already mentioned that the basis $\mathcal{Q}^{+}$in the case $n=1$ satisfies Stein's property. Therefore, using only the weak-type $(p, p)$ of $M^{+}$we have, exactly as above, both Sawyer's theorem and the property $A_{p}^{+} \Rightarrow$ $A_{p-\varepsilon}^{+}$. 
It turns out that the case $n \geq 2$ in the study of $M^{+}$is much more complicated. In fact, the question whether the full analogue of Sawyer's theorem holds when $n \geq 2$ is still open. Only in a recent paper [7], the authors overcame considerable technical difficulties and proved that in the case $n=2$ the $A_{p}^{+}$condition is equivalent to the weak type $(p, p)$ property of $M^{+}$. Observe that a dyadic variant of this result was recently obtained in [19] in any dimension. However, the usual, non-dyadic case requires much more delicate analysis, and it is unknown for us whether the covering argument found in $[\mathbf{7}]$ in the case $n=2$ can be extended to $n \geq 3$.

Once an equivalence between the weak type $(p, p)$ of $M^{+}$and the $A_{p}^{+}$condition is established, it is natural to ask whether the basis $\mathcal{Q}^{+}$, $n=2$, satisfies Stein's property, as in the one-dimensional case. Unfortunately, this is not true as the following example shows.

Example 1.4. Let $n=2$. Then $\mathcal{Q}^{+}$does not satisfy Stein's property.

Let $Q_{0}=(0,1)^{2}$ and $f_{\varepsilon}=\frac{1}{\varepsilon^{2}} \chi_{(0, \varepsilon) \times(1-\varepsilon, 1)}$ for small $\varepsilon$. It is easy to see that $M^{+} f_{\varepsilon}(0)=1$ and $\left\{y \in Q_{0}: M^{+} f_{\varepsilon}(y)>\lambda\right\} \subset(0, \varepsilon) \times(0,1)$. Hence, setting in (1.1) $f=f_{\varepsilon}$ and $B=Q_{0}$, for any fixed $\lambda$ such that $1<\lambda<\frac{1}{\varepsilon^{2}}$ we get that the left-hand side of (1.1) is equal to 1 , while the right-hand side is bounded by $c \lambda \varepsilon$.

Roughly speaking, Theorem 1.2 contains implicitly a large part of the standard technique needed to work with "good" maximal operators. The above example shows that this technique falls down when we deal with $M^{+}$in the multi-dimensional case. Nevertheless, some indirect variants of ideas used in proving Theorem 1.2 combined with the above mentioned weak type result for $M^{+}$proved in $[7]$ allow us to get a strong type result for a family of maximal operators closely related to $\mathrm{M}^{+}$. This family is defined as follows. Given $x=\left(x_{1}, x_{2}\right)$ and $r \in[0,1)$, let $Q_{x, h}^{r}=\prod_{i=1}^{2}\left(x_{i}+r h, x_{i}+h\right)$. For $f \in L_{\mathrm{loc}}^{1}\left(\mathbb{R}^{2}\right)$ define the maximal operator $N_{r}^{+}$by

$$
N_{r}^{+} f(x)=\sup _{h>0} \frac{1}{\left|Q_{x, h}^{r}\right|} \int_{Q_{x, h}^{r}}|f(y)| d y .
$$

Observe that $N_{0}^{+} f=M^{+} f$ and $N_{r_{2}}^{+} f \leq c N_{r_{1}}^{+} f$ for $0 \leq r_{1}<r_{2}<1$.

The second main result of this paper is the following.

Theorem 1.5. Let $1<p<\infty$. If $w \in A_{p}^{+}\left(\mathbb{R}^{2}\right)$, then

$$
\left\|N_{r}^{+} f\right\|_{L_{w}^{p}} \leq c\|f\|_{L_{w}^{p}} \quad(0<r<1),
$$

where the constant $c$ depends only on $w, p$ and $r$. 
It is easy to show that in the one-dimensional case $N_{r}^{+} f$ is equivalent to $M^{+} f$ (see, e.g., [16, Proposition 2.4]), and this is not true in general when $n \geq 2$. Hence, Theorem 1.5 can be regarded as an extension of Sawyer's theorem to the case $n=2$. Notice that the main question whether the $A_{p}^{+}\left(\mathbb{R}^{2}\right)$ condition is sufficient for the boundedness of $M^{+}$ on $L_{w}^{p}\left(\mathbb{R}^{2}\right)$ remains open. However, Theorem 1.5 shows that this really holds for an arbitrary big portion of $M^{+}$. This gives an additional indication that an answer to the above question should be positive.

The paper is organized as follows. Section 2 contains the proof of Theorem 1.2. Theorem 1.5 is proved in Section 3. Finally, in Section 4 we consider some other applications of Theorem 1.2.

Acknowledgements. This work was done during our stay at the University of Seville. We would like to thank Carlos Pérez for his hospitality.

We are grateful to the referee for useful comments and remarks.

\section{Proof of Theorem 1.2}

For the definition of Banach function norm we refer to [2, p. 2]. If the triangle inequality in this definition is replaced by $\|f+g\| \leq c(\|f\|+\|g\|)$ for some $c \geq 1$, we get a quasi-norm. A complete quasi-normed space is called a quasi-Banach space. We shall use the following version of the Aoki-Rolewicz theorem (see, e.g., [11, p. 3]) saying that for a quasiBanach space $X$,

$$
\left\|\sum_{k=0}^{\infty} f_{k}\right\|_{X} \leq 4^{1 / \rho}\left(\sum_{k=0}^{\infty}\left\|f_{k}\right\|_{X}^{\rho}\right)^{1 / \rho},
$$

where $0<\rho \leq 1$ is given by $c=2^{1 / \rho-1}$ ( $c$ is the "quasi-norm" constant).

We say that a weight $w$ satisfies the $A_{1}(\mathcal{B})$ condition if there exists $c>0$ such that

$$
M_{\mathcal{B}} w(x) \leq c w(x) \quad \text { a.e. }
$$

The smallest possible $c$ in $(2.2)$ is denoted by $\|w\|_{A_{1}(\mathcal{B})}$.

Lemma 2.1. Suppose $\mathcal{B}$ satisfies Stein's property. If $w \in A_{1}(\mathcal{B})$, then

$$
M_{\mathcal{B}, r} w(x) \leq 2\|w\|_{A_{1}(\mathcal{B})} w(x) \quad \text { a.e. },
$$

where $r=1+\frac{\xi}{\|w\|_{A_{1}(\mathcal{B})}}$, and $\xi$ depends only on the constant $c$ from Definition 1.1. 
Remark 2.2. When $\mathcal{B}=\mathcal{Q}$ this lemma was used in a recent paper $[\mathbf{1 2}]$ in order to get some sharp weighted inequalities for singular integrals. Note that actually the lemma is contained implicitly in $[4],[9]$ but the dependence of $r$ on $\|w\|_{A_{1}(\mathcal{B})}$ is not written there explicitly. Since this point will be important for us, we give a complete proof of the lemma, although the case of general $\mathcal{B}$ is treated exactly as $\mathcal{Q}$.

Proof of Lemma 2.1: Let $B \in \mathcal{B}(x)$. By Fubini's theorem,

$$
\begin{aligned}
\int_{B} w^{1+\delta} d y= & \delta \int_{M_{\mathcal{B}} w(x)}^{\infty} \lambda^{\delta-1} \int_{\{y \in B: w(y)>\lambda\}} w(y) d y d \lambda \\
& +\delta \int_{0}^{M_{\mathcal{B}} w(x)} \lambda^{\delta-1} \int_{\{y \in B: w(y)>\lambda\}} w(y) d y d \lambda .
\end{aligned}
$$

Further, by Stein's property

$$
\begin{aligned}
\delta \int_{M_{\mathcal{B}} w(x)}^{\infty} \lambda^{\delta-1} \int_{\{y \in B: w(y)>\lambda\}} w(y) d y d \lambda \\
\leq c \delta \int_{M_{\mathcal{B}} w(x)}^{\infty} \lambda^{\delta}\left|\left\{y \in B: M_{\mathcal{B}} w(y)>\lambda\right\}\right| d \lambda \\
\leq \frac{c \delta}{1+\delta} \int_{B}\left(M_{\mathcal{B}} w\right)^{1+\delta} d y \leq \frac{c \delta\|w\|_{A_{1}(\mathcal{B})}^{1+\delta}}{1+\delta} \int_{B} w^{1+\delta} d y .
\end{aligned}
$$

Next, we trivially have

$$
\begin{aligned}
\delta \int_{0}^{M_{\mathcal{B}} w(x)} \lambda^{\delta-1} \int_{\{y \in B: w(y)>\lambda\}} w(y) d y d \lambda & \leq M_{\mathcal{B}} w(x)^{\delta} \int_{B} w \\
& \leq|B| M_{\mathcal{B}} w(x)^{1+\delta} .
\end{aligned}
$$

Therefore,

$$
\int_{B} w^{1+\delta} d y \leq \frac{c \delta\|w\|_{A_{1}(\mathcal{B})}^{1+\delta}}{1+\delta} \int_{B} w^{1+\delta} d y+|B| M_{\mathcal{B}} w(x)^{1+\delta} .
$$

Setting $\delta=\frac{1}{3 \max (c, 1)} \frac{1}{\|w\|_{A_{1}(\mathcal{B})}}$, we get $\frac{c \delta\|w\|_{A_{1}(\mathcal{B})}^{1+\delta}}{1+\delta} \leq \frac{1}{3} e^{1 / 3 e} \leq \frac{1}{2}$, and thus

$$
\frac{1}{|B|} \int_{B} w^{1+\delta} d y \leq 2 M_{\mathcal{B}} w(x)^{1+\delta} \text {. }
$$

This proves the lemma with $r=1+\delta$. 
Proof of Theorem 1.2: Following the Rubio de Francia idea [20], for $0<$ $\varepsilon<1$ set

$$
R_{\varepsilon} f(x)=\sum_{k=0}^{\infty} \varepsilon^{k} M_{\mathcal{B}}^{k} f(x),
$$

where $M_{\mathcal{B}}^{k}$ is the operator $M_{\mathcal{B}}$ iterated $k$ times and $M_{\mathcal{B}}^{0} f=|f|$. Note that $R_{\varepsilon} f(x) \in A_{1}(\mathcal{B})$ with $\left\|R_{\varepsilon} f\right\|_{A_{1}(\mathcal{B})} \leq \frac{1}{\varepsilon}$. Also we trivially have $|f| \leq R_{\varepsilon} f$. Therefore, setting $w(x)=R_{\varepsilon} f(x)$ in (2.3) and using Hölder's inequality, we get

$$
M_{\mathcal{B}, 1+\xi \varepsilon} f(x) \leq \frac{2}{\varepsilon} R_{\varepsilon} f(x) \quad(0<\varepsilon<1) .
$$

Observe that only two implications in Theorem 1.2 are non-trivial, namely, (i) $\Rightarrow$ (ii) and (ii) $\Rightarrow$ (iii). To prove the last implication, we apply (2.1) and (2.4) with $\varepsilon<1 /\left\|M_{\mathcal{B}}\right\|_{X}$. Then

$$
\begin{aligned}
\left\|M_{\mathcal{B}, 1+\xi \varepsilon} f\right\|_{X} \leq \frac{2}{\varepsilon}\left\|R_{\varepsilon} f\right\|_{X} & \leq \frac{2}{\varepsilon} 4^{1 / \rho}\left(\sum_{k=0}^{\infty}\left(\varepsilon^{k}\left\|M_{\mathcal{B}}^{k} f\right\|_{X}\right)^{\rho}\right)^{1 / \rho} \\
& \leq \frac{2}{\varepsilon} 4^{1 / \rho}\left(\sum_{k=0}^{\infty}\left(\varepsilon\left\|M_{\mathcal{B}}\right\|_{X}\right)^{\rho k}\right)^{1 / \rho}\|f\|_{X},
\end{aligned}
$$

and thus we have (iii) with $r=1+\xi \varepsilon$.

The proof of (i) $\Rightarrow$ (ii) is similar. Given $\varepsilon>0$, set $\nu_{\varepsilon}=1+\xi \varepsilon$. Using (i), fix an $\varepsilon>0$ such that $\varepsilon\left\|M_{\mathcal{B}, 1 / \nu_{\varepsilon}}\right\|_{X}<1$. Denote by $X_{\varepsilon}$ the quasi-Banach space with quasi-norm

$$
\|f\|_{X_{\varepsilon}}=\left\||f|^{\nu_{\varepsilon}}\right\|_{X}^{\frac{1}{\nu_{\varepsilon}}}
$$

Rewriting (2.4) as

$$
M_{\mathcal{B}} f(x) \leq\left(\frac{2}{\varepsilon} R_{\varepsilon}\left(|f|^{\frac{1}{\nu_{\varepsilon}}}\right)(x)\right)^{\nu_{\varepsilon}}
$$


and applying (2.1) to $X=X_{\varepsilon}$ (with the corresponding constant $\rho=\rho_{\varepsilon}$ ), we get

$$
\begin{aligned}
\left\|M_{\mathcal{B}} f\right\|_{X} & \leq(2 / \varepsilon)^{\nu_{\varepsilon}}\left\|R_{\varepsilon}\left(|f|^{\frac{1}{\nu_{\varepsilon}}}\right)\right\|_{X_{\varepsilon}}^{\nu_{\varepsilon}} \\
& \leq(2 / \varepsilon)^{\nu_{\varepsilon}} 4^{\nu_{\varepsilon} / \rho_{\varepsilon}}\left(\sum_{k=0}^{\infty}\left(\varepsilon^{k}\left\|M_{\mathcal{B}}^{k}\left(|f|^{\frac{1}{\nu_{\varepsilon}}}\right)\right\|_{X_{\varepsilon}}\right)^{\rho_{\varepsilon}}\right)^{\nu_{\varepsilon} / \rho_{\varepsilon}} \\
& =(2 / \varepsilon)^{\nu_{\varepsilon}} 4^{\nu_{\varepsilon} / \rho_{\varepsilon}}\left(\sum_{k=0}^{\infty} \varepsilon^{\rho_{\varepsilon} k}\left\|M_{\mathcal{B}, 1 / \nu_{\varepsilon}}^{k} f\right\|_{X}^{\rho_{\varepsilon} / \nu_{\varepsilon}}\right)^{\nu_{\varepsilon} / \rho_{\varepsilon}} \\
& \leq(2 / \varepsilon)^{\nu_{\varepsilon}} 4^{\nu_{\varepsilon} / \rho_{\varepsilon}}\left(\sum_{k=0}^{\infty}\left(\varepsilon\left\|M_{\mathcal{B}, 1 / \nu_{\varepsilon}}\right\|_{X}\right)^{\rho_{\varepsilon} k}\right)^{\nu_{\varepsilon} / \rho_{\varepsilon}}\|f\|_{X} .
\end{aligned}
$$

We have obtained (ii), and therefore the theorem is proved.

\section{Proof of Theorem 1.5}

We first introduce some notation. Given a square $Q=(a, a+h) \times$ $(b, b+h)$, for $\xi>0$ set $\widetilde{Q}_{\xi}=(a-\xi h, a+h) \times(b-\xi h, b+h)$ and $Q_{\xi}^{-}=(a-\xi h, a) \times(b-\xi h, b)$ (see Figure 1). Let $Q^{-}=Q_{1}^{-}$. Denote $f_{Q}=\frac{1}{|Q|} \int_{Q} f$. Let $\ell_{Q}$ be the side length of $Q$. For a measurable set $E$, let $w(E)=\int_{E} w$.
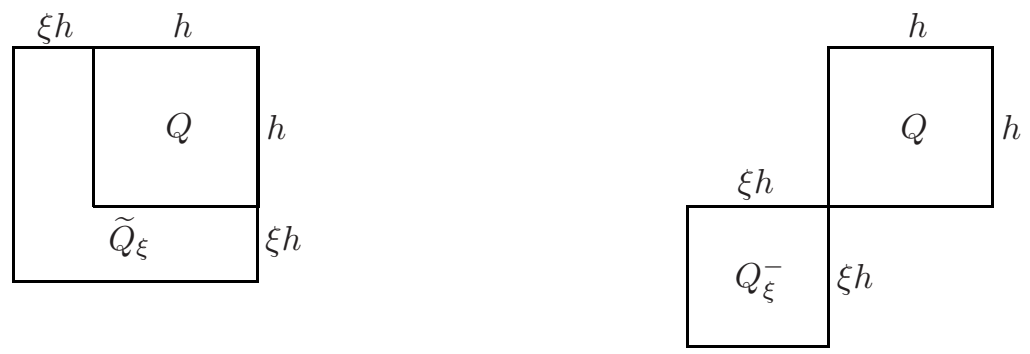

Figure 1. $\widetilde{Q}_{\xi}$ and $Q_{\xi}^{-}$.

As we mentioned in the Introduction, the proof of Theorem 1.5 contains some variants of ideas used in proving Theorem 1.2. The following lemma represents an analogue of Lemma 2.1. 
Lemma 3.1. There exists a constant $c>0$ such that for any weight $w$ and for any square $Q$,

$$
\int_{Q} w^{1+\delta} \leq c \frac{\delta}{\xi^{2}} \int_{\widetilde{Q}_{\xi}}\left(M^{+} w\right)^{1+\delta}+|Q|\left(w_{Q}\right)^{1+\delta} \quad(\delta>0,0<\xi \leq 1) .
$$

Proof: By Stein's estimate [23], for $\lambda>w_{Q}$,

$$
\int_{\{x \in Q: w(x)>\lambda\}} w(x) d x \leq 4 \lambda\left|\left\{x \in Q: M_{Q}^{\Delta} w(x)>\lambda\right\}\right|,
$$

where $M_{Q}^{\Delta}$ is the dyadic maximal function restricted to a square $Q$. From this, by Fubini's theorem we have,

$$
\begin{aligned}
\int_{\left\{x \in Q: w(x)>w_{Q}\right\}} w^{1+\delta} d x & =\delta \int_{w_{Q}}^{\infty} \lambda^{\delta-1} \int_{\{x \in Q: w(x)>\lambda\}} w(x) d x d \lambda \\
& \leq 4 \delta \int_{w_{Q}}^{\infty} \lambda^{\delta}\left|\left\{x \in Q: M_{Q}^{\Delta} w(x)>\lambda\right\}\right| d \lambda
\end{aligned}
$$

Let us show now that for $\lambda>w_{Q}$ and $0<\xi \leq 1$,

$$
\left|\left\{x \in Q: M_{Q}^{\Delta} w(x)>\lambda\right\}\right| \leq \frac{c}{\xi^{2}}\left|\left\{x \in \widetilde{Q}_{\xi}: M^{+} w(x)>\lambda / 4\right\}\right| .
$$

We have that $\left\{x \in Q: M_{Q}^{\Delta} w(x)>\lambda\right\}=\cup_{j} Q_{j}$, where $w_{Q_{j}}>\lambda$. For any point $x \in\left(Q_{j}\right)_{\xi}^{-}$there exists a square $Q_{j}^{\prime}$ containing $Q_{j}$ with $\left|Q_{j}^{\prime}\right| \leq$ $4\left|Q_{j}\right|$, and such that $x$ is the lower left corner of $Q_{j}^{\prime}$. It follows from this that $w_{Q_{j}^{\prime}} \geq \frac{1}{4} w_{Q_{j}}>\frac{\lambda}{4}$. Therefore, $M^{+} w(x)>\frac{\lambda}{4}$ for all $x \in\left(Q_{j}\right)_{\xi}^{-}$. Next, we note that $Q_{j} \subset\left(1+\frac{2}{\xi}\right)\left(Q_{j}\right)_{\xi}^{-}$. Applying the Vitali covering lemma (see, e.g., $\left[\mathbf{2}\right.$, p. 118]) to the family $\left\{\left(1+\frac{2}{\xi}\right)\left(Q_{j}\right)_{\xi}^{-}\right\}$we get pairwise disjoint squares $\left(1+\frac{2}{\xi}\right)\left(Q_{i}\right)_{\xi}^{-}, i=1, \ldots, k$ such that

$$
\begin{aligned}
\left|\bigcup_{j} Q_{j}\right| & \leq\left|\bigcup_{j}\left(1+\frac{2}{\xi}\right)\left(Q_{j}\right)_{\xi}^{-}\right| \\
& \leq 16 \sum_{i=1}^{k}\left|\left(1+\frac{2}{\xi}\right)\left(Q_{i}\right)_{\xi}^{-}\right|=16\left(1+\frac{2}{\xi}\right)^{2} \sum_{i=1}^{k}\left|\left(Q_{i}\right)_{\xi}^{-}\right| .
\end{aligned}
$$

Next we clearly have that the squares $\left(Q_{i}\right)_{\xi}^{-}, i=1, \ldots, k$ are also pairwise disjoint, and $\cup_{i=1}^{k}\left(Q_{i}\right)_{\xi}^{-} \subset\left\{x \in \widetilde{Q}_{\xi}: M^{+} w(x)>\lambda / 4\right\}$. From this and from (3.3) we get (3.2). 
Applying (3.1) and (3.2) gives

$$
\int_{\left\{x \in Q: w(x)>w_{Q}\right\}} w^{1+\delta} d x \leq c \frac{\delta}{\xi^{2}} \int_{\widetilde{Q}_{\xi}}\left(M^{+} w\right)^{1+\delta} d x
$$

from which the lemma follows easily.

The next lemma will be an important ingredient in proving the subsequent statement.

Lemma 3.2. Let $F$ be the convex hull of $Q_{\xi}^{-} \cup Q, \xi \geq 1$ (see Figure 2), and let $w \in A_{p}^{+}$. Then

$$
w(F) \leq c w(Q)
$$

where the constant $c$ depends only on $\xi, p$ and $w$.
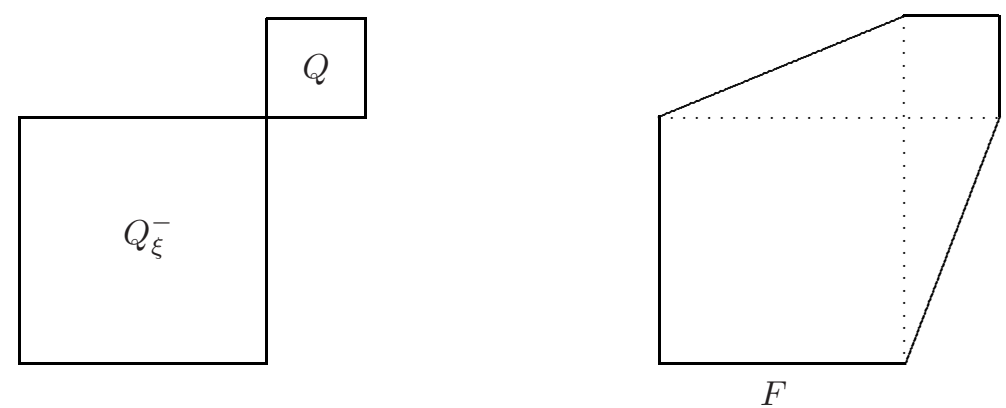

Figure 2. Convex hull.

Proof: When $\xi=1 / 4$ this was proved by F. J. Martín-Reyes [15]. In the general case the proof is similar but we give it for the sake of completeness.

We observe first that for any square $Q$,

$$
w\left(Q_{\xi}^{-}\right) \leq c w(Q) .
$$

Indeed, note that $Q \subset\left(Q_{\xi}^{-}\right)^{+}$. Therefore, setting $\sigma=w^{-1 /(p-1)}$ and applying the $A_{p}^{+}$condition along with Hölder's inequality, we get

$$
w\left(Q_{\xi}^{-}\right) \sigma\left(\left(Q_{\xi}^{-}\right)^{+}\right)^{p-1} \leq c \xi^{p}|Q|^{p} \leq c \xi^{p} w(Q) \sigma\left(\left(Q_{\xi}^{-}\right)^{+}\right)^{p-1},
$$

which proves (3.4).

Next we have that $F \backslash\left(Q_{\xi}^{-} \cup Q\right)$ is the union of two triangles $T_{1} \cup T_{2}$. In view of (3.4), it remains to show that $w\left(T_{i}\right) \leq c w(Q), i=1,2$. By symmetry, it suffices to consider the case $i=1$. 
Let $Q=(a, a+h) \times(b, b+h)$. Then it is easy to see that $T_{1}$ is covered (up to a set of measure zero) by $\cup_{j=0}^{\infty} Q_{j}$, where

$$
Q_{j}=\left(a-\frac{\xi h}{2^{j}}, a+\frac{h}{2^{j+1}}\right) \times\left(b+h-\frac{(1+\xi) h}{2^{j}}, b+h-\frac{h}{2^{j+1}}\right) .
$$

Next, $Q_{j}=\left(P_{j}\right)_{2 \xi+1}^{-}$, where

$$
P_{j}=\left(a+\frac{h}{2^{j+1}}, a+\frac{h}{2^{j}}\right) \times\left(b+h-\frac{h}{2^{j+1}}, b+h\right) .
$$

Clearly, $\cup_{j=0}^{\infty} P_{j} \subset Q$ and $P_{j}$ are pairwise disjoint. Hence, by (3.4),

$$
w\left(T_{1}\right) \leq \sum_{j=0}^{\infty} w\left(Q_{j}\right) \leq c \sum_{j=0}^{\infty} w\left(P_{j}\right) \leq c w(Q) .
$$

The proof is complete.

The following lemma is a key part of our proof.

Lemma 3.3. Let $w \in A_{p}^{+}$. Then

$w\left\{x: N_{r}^{+} f(x)>\lambda\right\} \leq c w\left\{x: N_{1 / 3}^{+} f(x)>\lambda / 3\right\} \quad(0<r<1 / 4, \lambda>0)$,

where the constant $c$ depends only on $r$ and $w$.

Proof: Set $E_{\lambda}=\left\{x: N_{r}^{+} f(x)>\lambda\right\}$, and let $x \in E_{\lambda}$. Then there exists $h>0$ such that $f_{Q_{x, h}^{r}}>\lambda$. Let $i=i(r)$ be the smallest natural number for which $2^{i} \geq 4 / r$. We divide $Q_{x, h}^{r}$ into $4^{i}$ equal squares. Then there exists at least one of them (denote it by $R_{x}$ ) such that $f_{R_{x}}>\lambda$.

Consider now the square $P_{x}=\left(R_{x}^{-}\right)^{-}$(see Figure 3 ). For any $y \in P_{x}$ there exists a square $\bar{Q}$ such that $y$ is the left lower corner of $\bar{Q}, R_{x} \subset$ $\bar{Q}_{y, \ell_{\bar{Q}}}^{1 / 3}$ and $|\bar{Q}| \leq 9\left|R_{x}\right|$. Then $f_{\bar{Q}_{y, \ell_{\bar{Q}}}^{1 / 3}} \geq(4 / 9) f_{R_{x}}>4 \lambda / 9$. Therefore, for any $y \in P_{x}$ we have $N_{1 / 3}^{+} f(y)>4 \lambda / 9$.

It is easy to see that there exists a square $P_{x}^{\prime}$ (see Figure 4 ) and such that

(i) the right upper corner of $P_{x}^{\prime}$ coincides with the left lower corner of $P_{x}$

(ii) $x \in \alpha P_{x}^{\prime}$, where $\alpha=\alpha(r)<1$;

(iii) $\ell_{P_{x}^{\prime}} \leq \beta \ell_{P_{x}}$, where $\beta=\beta(r)>1$.

Let $F_{x}$ be the convex hull of $P_{x}^{\prime} \cup P_{x}$. Applying to the family $\left\{F_{x}\right\}_{x \in E_{\lambda}}$ the Besicovitch covering theorem [8, Chapter 1$]$, we get a sequence $\left\{x_{k}\right\}$ such that

(i) $E_{\lambda} \subset \cup_{k} F_{x_{k}}$;

(ii) $\sum_{k} \chi_{F_{x_{k}}}(x) \leq c$. 


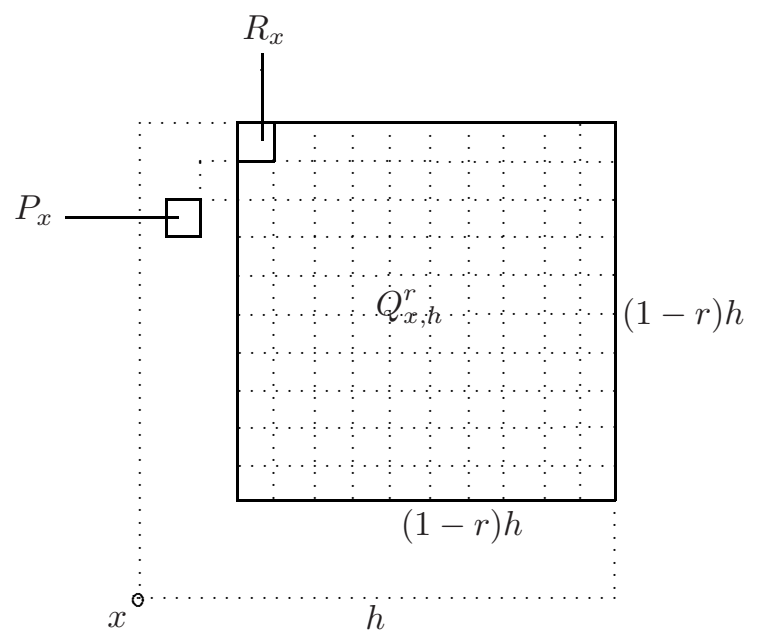

Figure 3

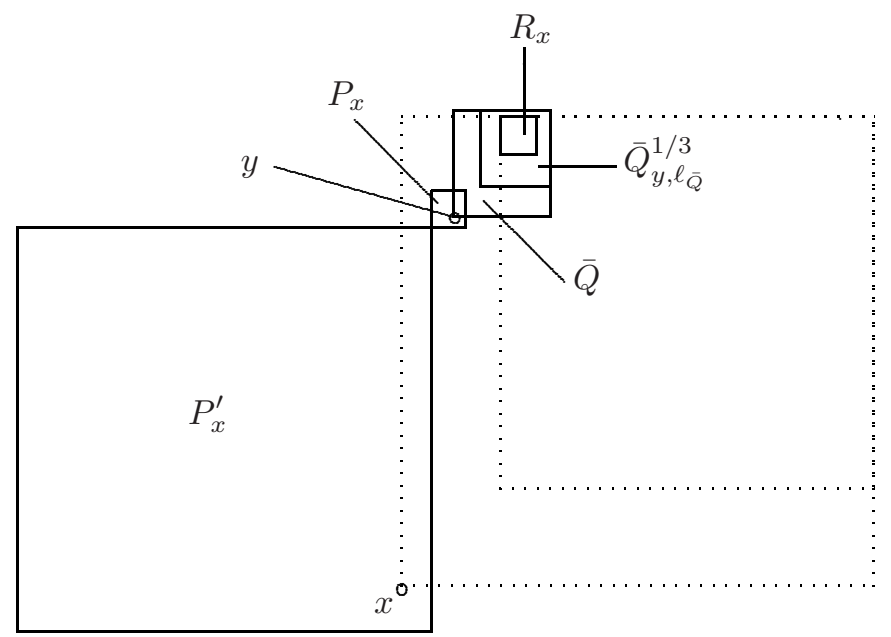

Figure 4

Therefore, by Lemma 3.2,

$w\left(E_{\lambda}\right) \leq \sum_{k} w\left(F_{x_{k}}\right) \leq c \sum_{k} w\left(P_{x_{k}}\right) \leq c w\left\{x: N_{1 / 3}^{+} f(x)>4 \lambda / 9\right\}$,

which completes the proof. 
Theorem 3.4. Let $n=2$. Then $M^{+}: L_{w}^{p} \rightarrow L_{w}^{p, \infty}$ if and only if $w \in$ $A_{p}^{+}$.

This theorem was proved in [7].

Proof of Theorem 1.5: One can assume that $0<r<1 / 4$. It follows from Lemma 3.1 that

$$
N_{1 / 3}^{+}\left(w^{1+\delta}\right)(x) \leq c \delta N_{r}^{+}\left(\left(M^{+} w\right)^{1+\delta}\right)(x)+\left(N_{1 / 3}^{+} w\right)^{1+\delta}(x),
$$

and therefore,

$$
N_{1 / 3}^{+}\left(w^{1+\delta}\right)(x) \leq c\|w\|_{A_{1}^{-}}^{1+\delta}\left(\delta N_{r}^{+}\left(w^{1+\delta}\right)(x)+w^{1+\delta}(x)\right)
$$

(here $A_{1}^{-}=A_{1}\left(\mathcal{Q}^{+}\right)$).

Let $R_{\varepsilon} f(x)=\sum_{k=0}^{\infty} \varepsilon^{k}\left(M^{+}\right)^{k} f(x)$. Then $\left\|R_{\varepsilon} f\right\|_{A_{1}^{-}} \leq \frac{1}{\varepsilon}$. Setting $w=R_{\varepsilon}\left(f^{\frac{1}{1+\delta}}\right)$ in (3.5), and denoting $T_{\varepsilon, \delta} f=R_{\varepsilon}\left(f^{\frac{1}{1+\delta}}\right)^{1+\delta}$, we get

$$
N_{1 / 3}^{+}\left(T_{\varepsilon, \delta} f\right)(x) \leq \frac{c}{\varepsilon^{1+\delta}}\left(\delta N_{r}^{+}\left(T_{\varepsilon, \delta} f\right)(x)+T_{\varepsilon, \delta} f(x)\right) .
$$

From this and from Lemma 3.3,

$$
\begin{aligned}
w\left\{x: N_{r}^{+}\left(T_{\varepsilon, \delta} f\right)(x)>\lambda\right\} \leq & c_{1} w\left\{x: N_{r}^{+}\left(T_{\varepsilon, \delta} f\right)(x)>\frac{\varepsilon^{1+\delta} \lambda}{6 c_{2} \delta}\right\} \\
& +c_{1} w\left\{x: T_{\varepsilon, \delta} f(x)>\frac{\varepsilon^{1+\delta} \lambda}{6 c_{2}}\right\} .
\end{aligned}
$$

Assume now that $f \in L^{\infty} \cap L_{w}^{p}$. Then $N_{r}^{+}\left(T_{\varepsilon, \delta} f\right) \in L^{\infty}$, and hence for any $a>0$,

$$
I(a)=\int_{a}^{\infty} \lambda^{p-1} w\left\{x: N_{r}^{+}\left(T_{\varepsilon, \delta} f\right)(x)>\lambda\right\} d \lambda<\infty .
$$

It follows from (3.6) that

$$
I(a) \leq c_{1}\left(\frac{6 c_{2} \delta}{\varepsilon^{1+\delta}}\right)^{p} I\left(a \varepsilon^{1+\delta} / 6 c_{2} \delta\right)+c(\varepsilon, \delta)\left\|T_{\varepsilon, \delta} f\right\|_{L_{w}^{p}}^{p} .
$$

Set now $\delta=\gamma \varepsilon$, where $\gamma$ is so that $c_{1}\left(\frac{6 c_{2} \gamma}{\varepsilon^{\gamma \varepsilon}}\right)^{p} \leq 1 / 2$. Then

$$
I(a) \leq 2 c(\varepsilon, \gamma \varepsilon)\left\|T_{\varepsilon, \gamma \varepsilon} f\right\|_{L_{w}^{p}}^{p} .
$$

Next we note that

$$
\left\|T_{\varepsilon, \gamma \varepsilon} f\right\|_{L_{w}^{p}} \leq\left(\sum_{k=0}^{\infty}\left(\varepsilon\left\|M^{+}\right\|_{\left.L_{w}^{p(1+\gamma \varepsilon)}\right)^{k}}\right)^{1+\gamma \varepsilon}\|f\|_{L_{w}^{p}} .\right.
$$


It follows from Theorem 3.4 and from the Marcinkiewicz interpolation theorem that

$$
\left\|M^{+}\right\|_{L_{w}^{p(1+\gamma \varepsilon)}} \leq \frac{c}{(\gamma \varepsilon)^{1 / p}} .
$$

Taking $\varepsilon$ so that $c \varepsilon^{1-1 / p} / \gamma^{1 / p}<1$, and combining the previous estimates, we obtain

$$
I(a) \leq c\|f\|_{L_{w}^{p}}^{p} .
$$

Letting $a \rightarrow 0$, and using that $|f| \leq T_{\varepsilon, \delta} f$, we get

$$
\left\|N_{r}^{+} f\right\|_{L_{w}^{p}} \leq c\|f\|_{L_{w}^{p}}
$$

Finally we note that the restriction $f \in L^{\infty}$ is easily removed by the Fatou convergence theorem.

\section{Some applications of Theorem 1.2}

4.1. Maximal characterization of the $A_{p}$ condition. Let

$$
M_{w} f(x)=\sup _{Q \ni x} \frac{1}{w(Q)} \int_{Q}|f(y)| w(y) d y .
$$

In the Introduction we have observed that Muckenhoupt's theorem follows easily from Corollary 1.3. The argument given shows that a weight $w$ satisfies the $A_{p}$ condition iff $w$ is doubling (i.e., there exists $c>0$ such that $w(2 Q) \leq c w(Q)$ for any $Q)$ and

$$
M f(x)^{p} \leq c M_{w}\left(|f|^{p}\right)(x) .
$$

Here we notice that the $A_{p}$ condition can be fully characterized in terms of (4.1) only.

Proposition 4.1. Let $w$ be a weight. Then $w$ satisfies the $A_{p}$ condition iff inequality (4.1) holds for any $f \in L_{\mathrm{loc}}^{1}\left(\mathbb{R}^{n}\right)$ and for all $x \in \mathbb{R}^{n}$.

Remark 4.2. The fact that (4.1) follows from the $A_{p}$ condition is wellknown [4]. However, we have never seen in the literature the converse statement.

Proof of Proposition 4.1: In the one-dimensional case the proof is immediate since the weighted maximal operator $M_{w}$ is always of weak type $(1,1)$ with respect to $w[\mathbf{2 2}]$, and therefore (4.1) implies the weak type $(p, p)$ of $M$. It remains to apply Corollary 1.3. In the case $n \geq 2$ we only need to show that (4.1) implies the doubling property of $w$. Then the same arguments work.

We shall use the notation from Section 3 with an obvious generalization to any dimension. First, we remark that for any cube $Q$,

$$
c_{1} w\left(Q_{\xi}^{-}\right) \leq w(Q) \leq c_{2} w\left(Q_{\xi}^{-}\right) \quad(\xi>0) .
$$


Indeed, let $x_{Q}$ be the "upper right" corner of $Q$. Then it is easy to see that with $f=\chi_{Q_{\xi}^{-}}$we have $M_{w}(f)\left(x_{Q}\right) \leq w\left(Q_{\xi}^{-}\right) / w(Q)$, and $M(f)\left(x_{Q}\right) \geq c$. From this and from (4.1) we get the right-hand side of (4.2); the left-hand side can be obtained in a similar way.

Next, observing that $Q_{1 / 2}^{-} \subset Q^{-}$, and combining inequalities in (4.2), we get

$$
w(2 Q) \leq c w\left((2 Q)^{-}\right) \leq c w\left(Q_{1 / 2}^{-}\right) \leq c w\left(Q^{-}\right) \leq c w(Q),
$$

which completes the proof.

4.2. On the property $A_{p}(\mathcal{B}) \Rightarrow A_{p-\varepsilon}(\mathcal{B})$. Let $\mathcal{B}$ be a BusemanFeller basis (BF-basis). This means that if $B \in \mathcal{B}$ and $x \in B$, then $B \in \mathcal{B}(x)$. Replacing in the definitions of $A_{p}$ and $M_{w}$ cubes by sets $B \in \mathcal{B}$ we get the $A_{p}(\mathcal{B})$ condition and the maximal operator $M_{\mathcal{B}, w}$. It is easy to see that the $A_{p}(\mathcal{B})$ condition is necessary for $M_{\mathcal{B}}$ to be bounded on $L_{w}^{p}$. Next, it was shown by B. Jawerth $[\mathbf{9}]$ that if

$$
A_{p}(\mathcal{B}) \Longrightarrow M_{\mathcal{B}, w}: L_{w}^{r} \longrightarrow L_{w}^{r} \quad(r>1),
$$

then $M_{\mathcal{B}}$ is bounded on $L_{w}^{p}$. Therefore, by (ii) $\Rightarrow$ (iii) of Theorem 1.2 we have that if $\mathcal{B}$ satisfies Stein's property and (4.3) holds, then $A_{p}(\mathcal{B}) \Rightarrow$ $A_{p-\varepsilon}(\mathcal{B})$.

Consider, for example, the Córdoba basis $\mathcal{R}_{\Phi}$, where $\mathcal{R}_{\Phi}(x)$ consists of all rectangles in $\mathbb{R}^{n}$ containing $x$ with dimensions $s_{1} \times \cdots \times s_{n-1} \times$ $\Phi\left(s_{1}, \ldots, s_{n-1}\right)$. Here $\Phi$ is a nonnegative continuous function, monotone in each variable and satisfying

$$
\Phi\left(s_{1}, \ldots, s_{j-1}, 0, s_{j+1}, \ldots, s_{n-1}\right)=0 \quad(1 \leq j \leq n-1),
$$

and $\Phi\left(s_{1}, \ldots, s_{n-1}\right) \approx \Phi\left(2 s_{1}, \ldots, 2 s_{n-1}\right)$. Clearly, $\mathcal{R}_{\Phi}$ is a BF-basis. Next, using properties of $\Phi$, it can be easily shown that $\mathcal{R}_{\Phi}$ satisfies Stein's property (it is enough to consider a "dyadic grid" with respect to a given rectangle $R$ and then use the same argument as in [23]). Finally, (4.3) for $\mathcal{B}=\mathcal{R}_{\Phi}$ was proved in [10]. Therefore, we have that $A_{p}\left(\mathcal{R}_{\Phi}\right) \Rightarrow A_{p-\varepsilon}\left(\mathcal{R}_{\Phi}\right)$. In the case $n=3$ and $\Phi(s, t)=s t$ this result is contained in $[\mathbf{6}]$.

4.3. Lorentz-Shimogaki Theorem. Given a measurable function $f$, the local maximal function $m_{\lambda} f$ is defined by

$$
m_{\lambda} f(x)=\sup _{Q \ni x}\left(f \chi_{Q}\right)^{*}(\lambda|Q|) \quad(0<\lambda<1),
$$

where $f^{*}$ denotes the non-increasing rearrangement of $f$. 
In a recent paper $[\mathbf{1 3}]$, the authors proved that the maximal operator $M$ is bounded on a quasi-Banach function space $X$ iff

$$
\alpha_{X} \equiv \lim _{\lambda \rightarrow 0} \frac{\log \left\|m_{\lambda}\right\|_{X}}{\log \frac{1}{\lambda}}<1 .
$$

This result is a generalization of the classical Lorentz-Shimogaki theorem [2, p. 154], since it is shown in [13] that in the case when $X$ is rearrangement-invariant the index $\alpha_{X}$ coincides with the upper Boyd index $\bar{\alpha}_{X}$.

As in the classical case, the part showing that the boundedness of $M$ implies $\alpha_{X}<1$ is more complicated. Among other ingredients, the proof in $[\mathbf{1 3}]$ was based on the theory of submultiplicative functions. Here we remark that this part follows immediately from Theorem 1.2. Indeed, by Chebyshev's inequality,

$$
\left(f \chi_{Q}\right)^{*}(\lambda|Q|)=\left(|f|^{r} \chi_{Q}\right)^{*}(\lambda|Q|)^{1 / r} \leq(1 / \lambda)^{1 / r}\left(\frac{1}{|Q|} \int_{Q}|f|^{r}\right)^{1 / r} .
$$

From this and from (ii) $\Rightarrow$ (iii) of Theorem 1.2 we get $\left\|m_{\lambda}\right\| \|_{X} \leq c(1 / \lambda)^{1 / r}$, and therefore $\alpha_{X} \leq 1 / r$ for some $r>1$.

4.4. Ariño-Muckenhoupt Theorem. Given a non-negative function $w$ on $(0, \infty)$, the Lorentz space $\Lambda_{p}(w)$ consists of all measurable $f$ on $\mathbb{R}^{n}$ for which

$$
\|f\|_{\Lambda_{p}(w)} \equiv\left(\int_{0}^{\infty} f^{*}(t)^{p} w(t) d t\right)^{1 / p}<\infty .
$$

In [1], M. A. Ariño and B. Muckenhoupt proved that $M$ is bounded on $\Lambda_{p}(w), 1 \leq p<\infty$, iff $w$ satisfies the following $B_{p}$ condition:

$$
\int_{t}^{\infty} \frac{w(\tau)}{\tau^{p}} d \tau \leq \frac{c}{t^{p}} \int_{0}^{t} w(\tau) d \tau \quad(t>0)
$$

Note that $(M f)^{*}(t) \asymp f^{* *}(t)=\frac{1}{t} \int_{0}^{t} f^{*}(\tau) d \tau$ [2, p. 122], and hence the boundedness of $M$ on $\Lambda_{p}(w)$ means that

$$
\left\|f^{* *}\right\|_{L_{w}^{p}} \leq c\left\|f^{*}\right\|_{L_{w}^{p}} .
$$

The key ingredient of the proof in [1] was the property $B_{p} \Rightarrow B_{p-\varepsilon}$. Later, C. J. Neugebauer [18] found a direct and simpler proof of (4.4); the property $B_{p} \Rightarrow B_{p-\varepsilon}$ was then deduced as a corollary.

Here we notice that exactly as in the case of $A_{p}$ weights, (ii) $\Rightarrow$ (iii) of Theorem 1.2 yields $B_{p} \Rightarrow B_{p-\varepsilon}$. In order to apply (ii) $\Rightarrow$ (iii) we only should mention the well-known fact saying that if $M$ is bounded 
on $\Lambda_{p}(w)$, then $\Lambda_{p}(w)$ is a Banach space (because the operator $f \rightarrow f^{* *}$ is subadditive [2, p. 53]).

For the sake of completeness we outline here a different elementary proof of the boundedness of $M$ on $\Lambda_{p}(w)$. Let $H \varphi(t)=\frac{1}{t} \int_{0}^{t} \varphi(\tau) d \tau$. Then the $B_{p}$ condition yields

$$
\begin{aligned}
\int_{0}^{\infty}(H \varphi)^{p}(t) w(t) d t & =\int_{0}^{\infty}(t H \varphi)^{p}(t)^{\prime} \int_{t}^{\infty} \frac{w(\tau)}{\tau^{p}} d \tau d t \\
& \leq c \int_{0}^{\infty}(t H \varphi)^{p}(t)^{\prime} \frac{1}{t^{p}} \int_{0}^{t} w(\tau) d \tau d t \\
& =c p \int_{0}^{\infty}\left(\int_{t}^{\infty}(H \varphi)^{p-1}(\tau) \frac{\varphi(\tau)}{\tau} d \tau\right) w(t) d t
\end{aligned}
$$

Let $\varphi(t)=f^{*}(t)-f^{*}(2 t)$. Then

$$
\begin{aligned}
\int_{t}^{\infty}(H \varphi)^{p-1}(\tau) \frac{\varphi(\tau)}{\tau} d \tau & \leq f^{* *}(t)^{p-1} \int_{t}^{\infty} \frac{f^{*}(\tau)-f^{*}(2 \tau)}{\tau} d \tau \\
& \leq f^{* *}(t)^{p-1} f^{*}(t),
\end{aligned}
$$

and applying (4.5) gives

$$
\int_{0}^{\infty}\left(f^{* *}(t)-f^{* *}(2 t)\right)^{p} w(t) d t \leq c \int_{0}^{\infty} f^{* *}(t)^{p-1} f^{*}(t) w(t) d t .
$$

Hence, using that $f^{* *}(t)-f^{*}(t) \leq 2\left(f^{* *}(t)-f^{* *}(2 t)\right)$, we get

$$
\left\|f^{* *}\right\|_{L_{w}^{p}} \leq\left\|f^{* *}-f^{*}\right\|_{L_{w}^{p}}+\left\|f^{*}\right\|_{L_{w}^{p}} \leq c\left(\int_{0}^{\infty} f^{* *}(t)^{p-1} f^{*}(t) w(t) d t\right)^{1 / p} .
$$

From this and Hölder's inequality we obtain (4.4).

We refer to a recent work [3] for numerous extensions and variants of the Ariño-Muckenhoupt theorem.

\section{References}

[1] M. A. Ariño and B. Muckenhoupt, Maximal functions on classical Lorentz spaces and Hardy's inequality with weights for nonincreasing functions, Trans. Amer. Math. Soc. 320(2) (1990), $727-735$.

[2] C. Bennett and R. Sharpley, "Interpolation of operators", Pure and Applied Mathematics 129, Academic Press, Inc., Boston, MA, 1988. 
[3] M. J. Carro, J. A. Raposo, and J. Soria, Recent developments in the theory of Lorentz spaces and weighted inequalities, Mem. Amer. Math. Soc. 187(877) (2007), 128 pp.

[4] R. R. Coifman and C. Fefferman, Weighted norm inequalities for maximal functions and singular integrals, Studia Math. 51 (1974), 241-250.

[5] J. DuonndikoetxeA, "Fourier analysis", Translated and revised from the 1995 Spanish original by David Cruz-Uribe, Graduate Studies in Mathematics 29, American Mathematical Society, Providence, RI, 2001.

[6] R. Fefferman, Some weighted norm inequalities for Córdoba's maximal function, Amer. J. Math. 106(5) (1984), 1261-1264.

[7] L. Forzani, F. J. Martín-Reyes, And S. Ombrosi, Weighted inequalities for the two-dimensional one-sided Hardy-Littlewood maximal function, Preprint, available at http: //webpersonal . uma.es/ MARTIN_REYES/preprints.htm.

[8] M. DE GuzMán, "Differentiation of integrals in $R^{n}$ ", with appendices by Antonio Córdoba, and Robert Fefferman, and two by Roberto Moriyón, Lecture Notes in Mathematics 481, SpringerVerlag, Berlin-New York, 1975.

[9] B. JAWERTH, Weighted inequalities for maximal operators: linearization, localization and factorization, Amer. J. Math. 108(2) (1986), 361-414.

[10] B. JaWERTh And A. Torchinsky, The strong maximal function with respect to measures, Studia Math. 80(3) (1984), 261-285.

[11] N. J. Kalton, N. T. Peck, and J. W. Roberts, "An F-space sampler", London Mathematical Society Lecture Note Series 89, Cambridge University Press, Cambridge, 1984.

[12] A. K. Lerner, S. Ombrosi, and C. PÉrez, Sharp $A_{1}$ bounds for Calderón-Zygmund operators and the relationship with a problem of Muckenhoupt and Wheeden, Int. Math. Res. Not. IMRN 6 (2008), Art. ID rnm161, 11 pp.

[13] A. K. Lerner And C. PÉREz, A new characterization of the Muckenhoupt $A_{p}$ weights through an extension of the Lorentz-Shimogaki theorem, Indiana Univ. Math. J. 56(6) (2007), 2697-2722.

[14] F. J. Martín-Reyes, New proofs of weighted inequalities for the one-sided Hardy-Littlewood maximal functions, Proc. Amer. Math. Soc. 117(3) (1993), 691-698.

[15] F. J. Martín-Reyes, On the one-sided Hardy-Littlewood maximal function in the real line and in dimensions greater than one, in: "Fourier analysis and partial differential equations" (Miraflores de 
la Sierra, 1992), Stud. Adv. Math., CRC, Boca Raton, FL, 1995, pp. $237-250$.

[16] F. J. Martín-Reyes And A. DE LA Torre, Two weight norm inequalities for fractional one-sided maximal operators, Proc. Amer. Math. Soc. 117(2) (1993), 483-489.

[17] B. Muckenhoupt, Weighted norm inequalities for the Hardy maximal function, Trans. Amer. Math. Soc. 165 (1972), 207-226.

[18] C. J. Neugebauer, Weighted norm inequalities for averaging operators of monotone functions, Publ. Mat. 35(2) (1991), 429-447.

[19] S. Ombrosi, Weak weighted inequalities for a dyadic one-sided maximal function in $\mathbb{R}^{n}$, Proc. Amer. Math. Soc. 133(6) (2005), 1769-1775 (electronic).

[20] J. L. Rubio DE Francia, Factorization theory and $A_{p}$ weights, Amer. J. Math. 106(3) (1984), 533-547.

[21] E. SAWYER, Weighted inequalities for the one-sided Hardy-Littlewood maximal functions, Trans. Amer. Math. Soc. 297(1) (1986), 53-61.

[22] P. SuÖGREn, A remark on the maximal function for measures in $\mathbb{R}^{n}$, Amer. J. Math. 105(5) (1983), 1231-1233.

[23] E. M. Stein, Note on the class L log L, Studia Math. 32 (1969), $305-310$.

Andrei K. Lerner:

Department of Mathematics

Bar-Ilan University

52900 Ramat Gan

Israel

E-mail address: aklerner@netvision.net.il

Sheldy Ombrosi:

Departamento de Matemática

Universidad Nacional del Sur

Bahía Blanca 8000

Argentina

E-mail address: sombrosi@uns.edu.ar

Primera versió rebuda el 27 de maig de 2008, darrera versió rebuda el 22 de juliol de 2009. 